\title{
Understanding spectroscopic phonon-assisted defect features in CVD grown 3C-SiC/Si( 100$)$ by modeling and simulation
}

\author{
Devki N. Talwar ${ }^{\mathrm{a}, *}$, Z.C. Feng ${ }^{\mathrm{b}}$ \\ a Department of Physics, Indiana University of Pennsylvania, 975 Oakland Avenue, Indiana, PA 15705-1087, USA \\ ${ }^{\mathrm{b}}$ Graduate Institute of Electro-Optical Engineering, National Taiwan University, Taipei 106, Taiwan, ROC
}

Received 12 December 2003; accepted 9 February 2004

\begin{abstract}
New phonon-assisted defect features are observed using photoluminescence (PL) and Raman scattering spectroscopy on $3 \mathrm{C}-\mathrm{SiC} / \mathrm{Si}(100)$ films grown by chemical vapor deposition (CVD) technique. The ultraviolet excitation roomtemperature (RT) PL-Raman spectra show a luminescence band near $2.3 \mathrm{eV}$ due to RT recombination over the 3C-SiC indirect band gap. In addition to the strong Raman lines characteristic of $\mathrm{Si}$ substrate and 3C-SiC we also observed weaker impurity modes near 620,743 and $833 \mathrm{~cm}^{-1}$. These frequencies are compared with the results of Green's function simulations of impurity modes with plausible defect structures to best support the observed Raman features as well as modes of some prototypical defect center.
\end{abstract}

(c) 2004 Elsevier B.V. All rights reserved.

Keywords: 3C-SiC, Raman scattering; Green function; Simulation

\section{Introduction}

Silicon carbide ( $\mathrm{SiC})$, owing to its excellent electrical, mechanical and chemical properties, is experiencing a renewed wave of interest in electronic applications with feasibility of use in extreme environments [1-4]. Continuing progress in the growth of bulk and epitaxial $\mathrm{SiC}$ films with defined doping levels and device processing has resulted in successful fabrication of several elec-

\footnotetext{
${ }^{*}$ Corresponding author. Fax: +1-724-3573804.

E-mail address: talwar@iup.edu (D.N. Talwar).
}

tronic devices with the same quality standards as of the Si technology.

Chemical vapor deposition (CVD) has been a successful and reliable method for the epitaxial growth of $\mathrm{SiC}$ films on $\mathrm{Si}$ and $\mathrm{SiC}$ substrates. Despite significant differences in the lattice constants $(\sim 20 \%)$ and thermal expansion coefficients $(\sim 8 \%)$ between $3 \mathrm{C}-\mathrm{SiC}$ and $\mathrm{Si}$, large area heteroepitaxial 3C-SiC/Si(100) layers are successfully grown by CVD technique [5-8]. The large lattice mismatch and high deposition temperature required for the growth of $\mathrm{SiC}$ films are primarily responsible for the fundamental material related problems including the residual strain, thermal 
expansion coefficient, low energy defect formation etc. The epitaxially grown films are usually n-type with high donor concentrations [9-11]. Recent studies by scanning electron microscopy (SEM) have revealed high density of lattice defects such as stacking faults and anti-phase boundaries [12,13]. As the reliability of semiconductor devices is strictly correlated to the presence of defects, the key to a successful SiC device technology demands (i) the control of doping, (ii) the knowledge of defect states within the material influencing the lifetime of devices and (iii) the site-selectivity of both intrinsic and doped defects.

The purpose of the present paper is to report the results of an extensive spectroscopic (photoluminescence (PL) and Raman scattering) investigations on $3 \mathrm{C}-\mathrm{SiC} / \mathrm{Si}\left(\begin{array}{lll}1 & 0 & 0\end{array}\right)$ films grown by $\mathrm{CVD}$ technique (cf. Section 2). We have performed both ultraviolet (UV) excitation (325 nm) PL-Raman measurements in the backscattering geometry [14] and Raman scattering with an excitation of 406.7 $\mathrm{nm}$ line from a $\mathrm{Kr}$ ion laser [15]. The room-temperature (RT) PL-Raman spectra exhibited a luminescence line near $2.3 \mathrm{eV}$ due to $\mathrm{RT}$ recombination over the $3 \mathrm{C}-\mathrm{SiC}$ indirect band gap. In addition to the strong Raman lines characteristic of $\mathrm{Si}$ substrate and 3C-SiC we have also observed weaker impurity modes near 620,743 and $833 \mathrm{~cm}^{-1}$. These frequencies are compared with the results of Green's function simulations (cf. Section 3) of impurity modes with plausible defect structures to best support the observed Raman features as well as the modes of some prototypical defect center.

\section{Experimental results}

The 3C-SiC samples used in the present spectroscopic studies are epitaxially grown on $\mathrm{Si}\left(\begin{array}{lll}1 & 0 & 0\end{array}\right)$ substrate by CVD method. The CVD 3C-SiC/Si films are generally reported to be under tensile strain. Measurements on the sample curvature have revealed that the sign of internal stress becomes abruptly negative when the initial stage of carbonization of $\mathrm{Si}$ substrate prior to growth is carried out at temperatures lower than $1050{ }^{\circ} \mathrm{C}$.

Our CVD method includes the preparation of an initial $\mathrm{SiC}$ layer from carbonization and sub- sequent growth of $\mathrm{SiC}$. The details of the growth process are described elsewhere [6]. It has been shown that the initial growth of 1-3 $\mu \mathrm{m}$ layer contained high density of dislocations, stacking fault, micro-cracking etc. After a certain thickness of growth, a very good and high quality $3 \mathrm{C}-\mathrm{SiC} /$ $\operatorname{Si}\left(\begin{array}{lll}1 & 0 & 0\end{array}\right)$ layers are obtained.

\subsection{Combined PL-Raman spectroscopy under $U V$ excitation}

In an earlier study Shim et al. [16] reported that an indirect band gap 3C-SiC/Si material can not exhibit luminescence at room temperature. If excitation comes from the interface side of the free 3C-SiC film (etching away Si substrate) a broad PL band near $2.4 \mathrm{eV}$ with a weak line at $3.0 \mathrm{eV}$ is observed. The authors of Ref. [16] speculated the origin of these bands as either due to recombination center associated with the $\mathrm{OH}$ group or localized states near the interface.

On the contrary by using a sensitive Renishaw UV Raman-PL microscope system we have realized a direct detection of room-temperature PL spectra in a $16 \mu \mathrm{m}$ 3C-SiC/Si(1 00$)$ CVD sample. The results displayed in Fig. 1a for the energy range $2.0-3.75 \mathrm{eV}$ reveal a band near $2.3 \mathrm{eV}$ originating from $3 \mathrm{C}-\mathrm{SiC}-\mathrm{a}$ representative of the PL emissions across the indirect band gap. The Raman scattering features (a sharp band $\sim 3.7 \mathrm{eV}$ and a weak line near $\sim 3.62 \mathrm{eV}$ ) are also visible. Raman shift plotted in Fig. 1b has clearly revealed the 3C-SiC longitudinal optical (LO) phonon at $\Gamma$ point $\left(\sim 980 \mathrm{~cm}^{-1}\right.$ with a FWHM of $14 \mathrm{~cm}^{-1}$ ) along with second-order Raman features between the energy range of $1500-1800 \mathrm{~cm}^{-1}$. The perusal of Fig. 1a reveals that our $2.3 \mathrm{eV}$ PL line of $3 \mathrm{C}-\mathrm{SiC} / \mathrm{Si}\left(\begin{array}{lll}1 & 0 & 0\end{array}\right)$ has a full width at half maximum (FWHM) of about $0.11 \mathrm{eV}$-much narrower ( $\sim 6$ times) than reported in Ref. [16]. This indicates the high quality of $3 \mathrm{C}$-SiC film from our CVD samples. The Raman scattering signals are generally much weaker than the PL emission. However the combined PL-Raman spectrum in Fig. 1a exhibiting Raman mode intensities at the same scale with the PL lines is a further testimony of the high crystalline quality of the $3 \mathrm{C}-\mathrm{SiC} / \mathrm{Si}$ materials. 

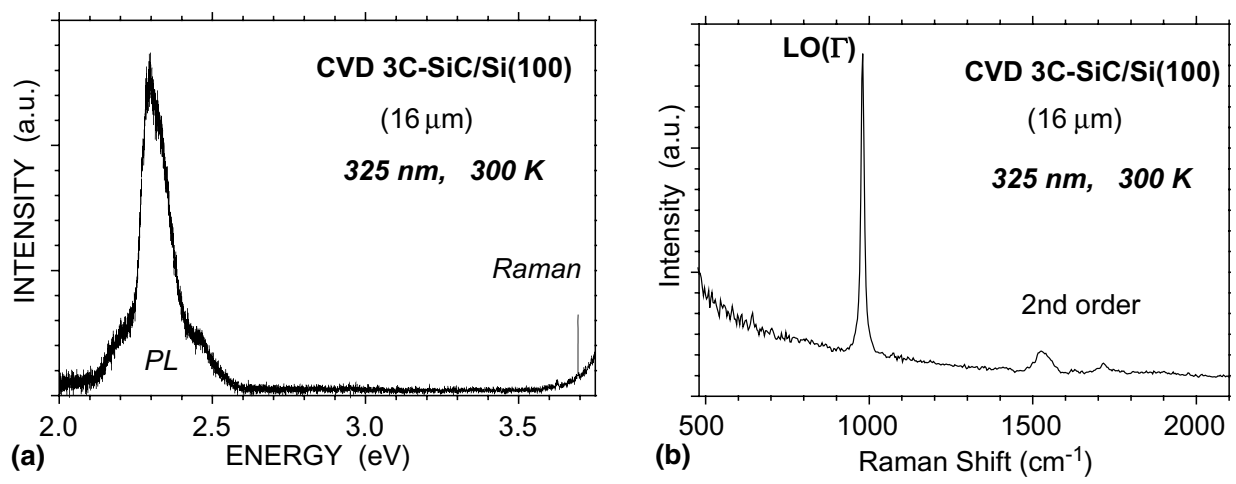

Fig. 1. Combined PL and Raman spectra, under UV (325 nm) excitation from a CVD 3C-SiC/Si(1 00$)$ sample with film thickness of 16 $\mu \mathrm{m}$ : (a) PL-Raman is displayed in the energy range of 2.0-3.75 eV and (b) Raman shift is plotted in wave number.

\subsection{Raman spectra}

In addition to the Raman active $3 \mathrm{C}-\mathrm{SiC}$ modes, we have observed new phonon-features in many 3C-SiC/Si( 100$)$ samples. In Fig. 2 the results of Raman scattering are displayed from a CVD 3C-

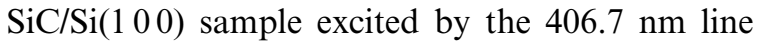
from a $\mathrm{Kr}^{+}$laser at $300 \mathrm{~K}$. Clearly, the strong Raman lines characteristic of Si substrate $[\mathrm{TO}(\Gamma)$ mode $]$ and 3C-SiC $[\mathrm{LO}(\Gamma)$ mode] are observed. By magnifying the intensity 50 times the weaker modes at 620,743 and $833 \mathrm{~cm}^{-1}$ become visible. In Fig. 3 we have plotted Raman spectra from three

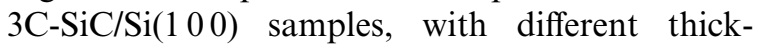
nesses. Two modes at 620 and $833 \mathrm{~cm}^{-1}$ are observed from the thinnest film of $4 \mu \mathrm{m}$ (cf. Fig. 3a). In the $7 \mu \mathrm{m}$ 3C-SiC/Si sample, besides the 620

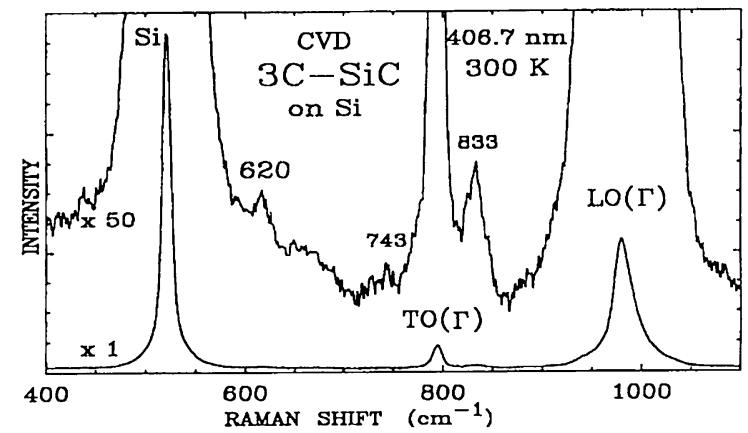

Fig. 2. Room-temperature Raman scattering from a CVD 3C$\mathrm{SiC} / \mathrm{Si}\left(\begin{array}{ll}1 & 0\end{array}\right)$ excited under $406.7 \mathrm{~nm}$. If magnified 50 times the weaker modes at 620,743 and $833 \mathrm{~cm}^{-1}$ are clearly observed. $\mathrm{cm}^{-1}$ mode, additional line at $\sim 680 \mathrm{~cm}^{-1}$ is seen. However, for the thickest $16 \mu \mathrm{m}$ film, the features at 620,680 and $833 \mathrm{~cm}^{-1}$, are not so obvious. Comprehensive Green's function simulations (cf. Section 3) of impurity modes with plausible defect structures are performed to best correlate the phonon-assisted Raman features to defects and to prototypical center.

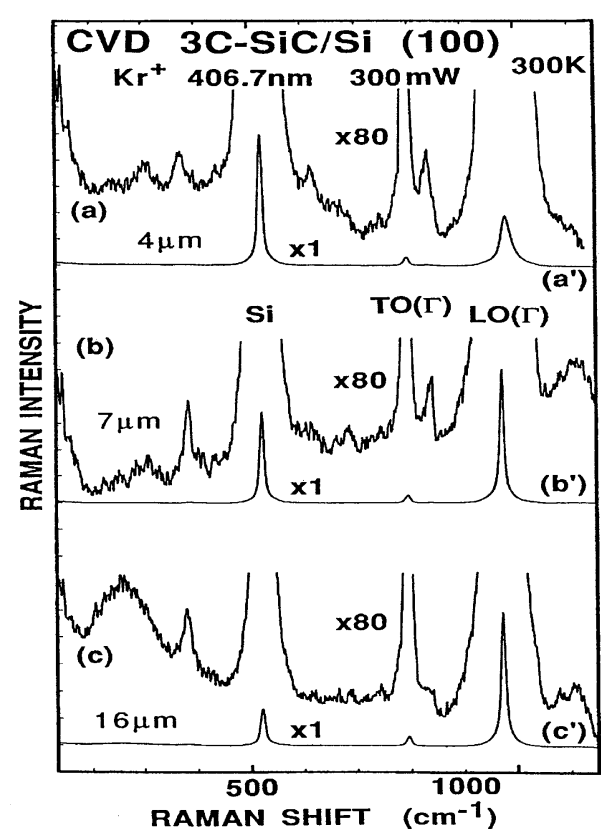

Fig. 3. Room-temperature Raman spectra from three CVD 3C$\mathrm{SiC} / \mathrm{Si}\left(\begin{array}{lll}1 & 0 & 0\end{array}\right)$ samples of different thickness. 


\section{Theoretical}

\subsection{Dynamical properties of defects in 3C-SiC}

The study of localized vibrational modes (LVMs) in compound semiconductors has provided us with valuable information about the siteselectivity and chemical nature of defects. Except for H-related modes the LVM data for intrinsic or extrinsic defects in $3 \mathrm{C}-\mathrm{SiC}$ is rather sparse. Green's function theory is used to study the impurity modes of both isolated and complex defects in 3C$\mathrm{SiC}$. The results are evaluated for plausible defect structures to best support the observed Raman features as well as a prototypical center.

\subsubsection{Isolated single substitutional defects}

In zinc-blende type materials the light impurities occupying either the cation or anion site vibrates in the triply degenerate $F_{2}$ mode causing high frequency infrared and Raman active LVMs. The heavier impurity, with appropriate 'impurity-host interactions', may give rise to Raman active $\mathrm{A}_{1}, \mathrm{E}$ and $F_{2}$ type 'quasi-localized' modes. Earlier, we have analyzed LVMs due to several isolated defects in III-V compounds and established valuable trends in the force-constant variations relating them to the redistribution of electron charge density in the impurity-host bonding. These trends in the bonding mechanism are important to establish the role of intrinsic and extrinsic defects in $3 \mathrm{C}-\mathrm{SiC}$.
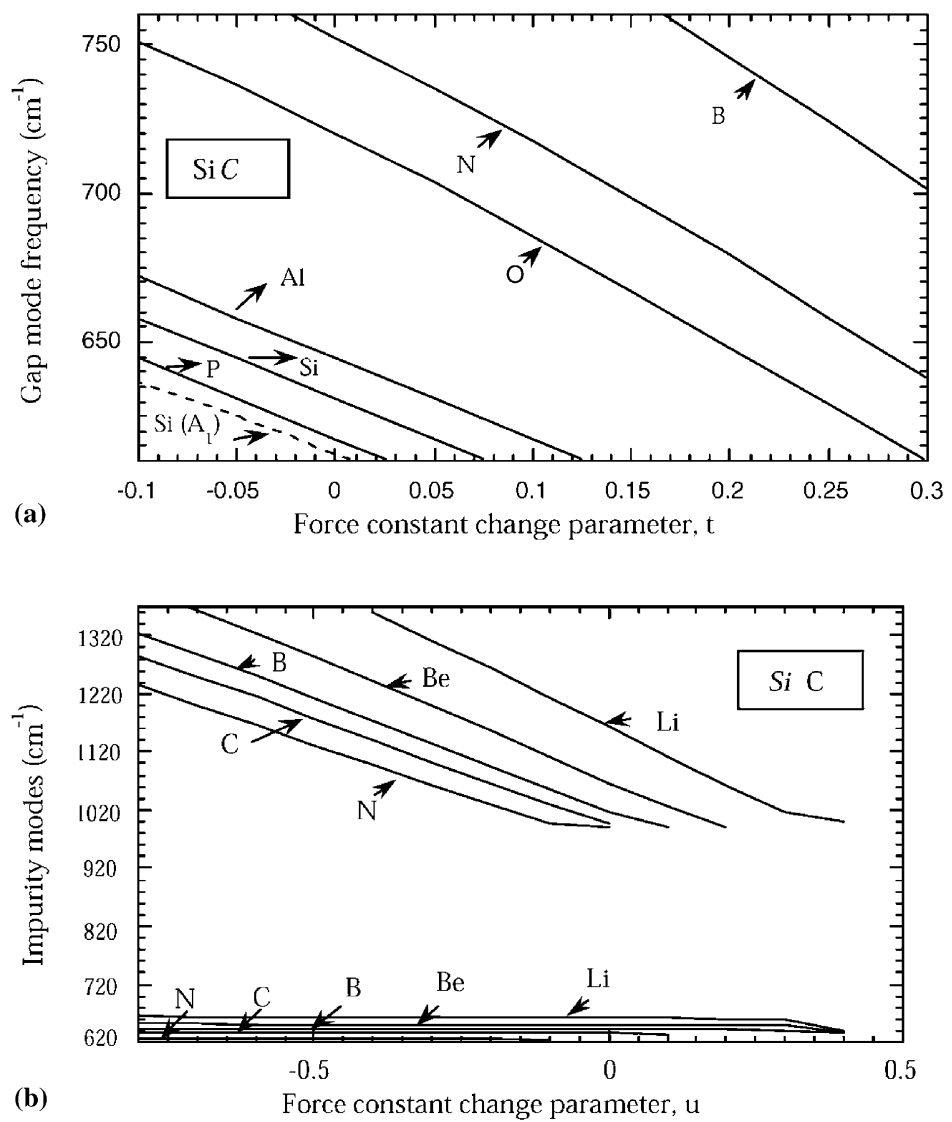

Fig. 4. (a) Green's function calculations of the gap modes as a function of force-constant change parameter $t$ for impurities occupying the $\mathrm{C}$ site in 3C-SiC. (b) Green's function calculations of the impurity (localized and gap) modes as a function of force-constant change parameter $u$ for defects occupying the $\mathrm{Si}$ site in $3 \mathrm{C}-\mathrm{SiC}$. 
In the Green's function frame work [17] we have simulated local and gap modes as a function of force-constant parameter $t(u)$ for several defects occupying Si or $\mathrm{C}$ sites in $3 \mathrm{C}-\mathrm{SiC}$ (see: Fig. $4 \mathrm{a}-\mathrm{b}$ ). The light impurities on Si-sites not only provide high frequency LVMs but also gap modes that emerge from the top of the acoustical branch (see: Fig. 4a). In addition to the 'quasi-localized' modes for heavy impurities on C-sites, our simulation predict $\mathrm{F}_{2}$-type gap modes emerging from the minimum of the optical band (see: Fig. 4b).

If the appropriate force-constant variations are included our simulations predicted local, gap modes for $\mathrm{B}_{\mathrm{Si}}\left(\sim 990 \mathrm{~cm}^{-1}, \sim 620 \mathrm{~cm}^{-1}\right), \mathrm{C}_{\mathrm{S}}(\sim 1000$ $\left.\mathrm{cm}^{-1}, 620 \mathrm{~cm}^{-1}\right), \mathrm{N}_{\mathrm{Si}}\left(\sim 985 \mathrm{~cm}^{-1}, \sim 620 \mathrm{~cm}^{-1}\right)$ and gap modes for $\mathrm{N}_{\mathrm{C}}\left(\sim 638 \mathrm{~cm}^{-1} \mathrm{~F}_{2}\right)$, and $\mathrm{Si}_{\mathrm{C}}(\sim 648$ $\left.\mathrm{cm}^{-1} \mathrm{~F}_{2} ; \sim 622 \mathrm{~cm}^{-1} \mathrm{~A}_{1}\right)$. Although the calculated impurity are infrared and Raman active most of them fall close to the phonon continuum of $3 \mathrm{C}$ SiC. This might be the reason why no such vibrations are detected by optical spectroscopy. Again, despite the relatively high frequency of the $\mathrm{C}$-antisite $\mathrm{C}_{\mathrm{Si}}\left(\sim 1000 \mathrm{~cm}^{-1}\right)$ mode it is not observed experimentally because the center is both electrically and optically inactive.

\subsubsection{Nearest-neighbor pair defects}

A prototypical $\mathrm{D}_{\mathrm{I}}$ defect center in $\mathrm{SiC}$ responsible for the sharp emission lines in the low temperature photoluminescence (LTPL) is well known for decades. The $\mathrm{D}_{\mathrm{I}}$ is observed in as-grown material after quenching from growth temperature as well as in epitaxial layers grown by CVD or molecular beam epitaxy (MBE) methods. The sharp $L_{i}$ emission lines are followed by characteristic phononassisted structures around $83 \mathrm{meV}\left(\sim 664 \mathrm{~cm}^{-1}\right)$ in the phonon band gap. These facts suggests that $D_{I}$ center has an intrinsic nature and is formed by vacancies, antisites and/or interstitials introduced by the damage. Several models have been proposed for the $D_{I}$ center including a divacancy; carbon divacancy; $\mathrm{V}_{\mathrm{Si}}-\mathrm{N}_{\mathrm{C}}$ complex, etc. Using Greens function theory we have calculated impurity modes for several nearest-neighbor pair defects $\left(\mathrm{V}_{\mathrm{Si}}-\mathrm{N}_{\mathrm{C}}\right.$; $\mathrm{Si}_{\mathrm{C}}-\mathrm{C}_{\mathrm{si}}$ ) of $\mathrm{C}_{3 \mathrm{v}}$ symmetry in $3 \mathrm{C}-\mathrm{SiC}$.

As reported in Section 3.1.1 the LVM of an isolated $\mathrm{C}_{\mathrm{Si}}$ defect $\left(\sim 1000 \mathrm{~cm}^{-1}\right)$ falls close to the maximum phonon frequency $\omega_{\mathrm{m}}$ of $3 \mathrm{C}-\mathrm{SiC}$, while the isolated $\mathrm{Si}_{\mathrm{C}}$ defect provides two impurity modes in the phonon gap - a triply degenerate $F_{2}$ mode $\left(\sim 648 \mathrm{~cm}^{-1}\right)$ and a non-degenerate $\mathrm{A}_{1}$ mode $(\sim 622$ $\mathrm{cm}^{-1}$ ) (see: Fig. 4a). The pairing of antisite defects $\mathrm{Si}_{\mathrm{C}}-\mathrm{C}_{\mathrm{Si}}$ lifts the degeneracies of the $\mathrm{F}_{2}$ modes. With an appropriate stiffening between the impurity bond the $\mathrm{F}_{2}$ gap mode splits up into two modes $\sim 651$ $\mathrm{cm}^{-1}\left(\mathrm{~A}_{1}\right)$ and $\sim 647 \mathrm{~cm}^{-1}$ (E). These results compare favorably well with our Raman data and are consistent with the observed characteristic phononassisted structure in the phonon gap of the $\mathrm{D}_{\mathrm{I}}$ center.

\section{Summary and conclusions}

In summary, we have carried out a comprehensive theoretical study to understand the spectroscopic phonon-assisted defect features observed in CVD grown $3 \mathrm{C}-\mathrm{SiC} / \mathrm{Si}\left(\begin{array}{lll}1 & 0 & 0\end{array}\right)$ samples. Our UV (325 nm) excitation PL-Raman spectra exhibited $2.3 \mathrm{eV}$ luminescence band due to RT recombination over the 3C-SiC indirect band gap. Raman scattering studies are made with an excitation of $406.7 \mathrm{~nm}$ from a $\mathrm{Kr}$ ion laser, in the back scattering geometry using a triple spectrometer. In addition to the strong Raman lines characteristic of Si substrate and 3C-SiC new impurity-activated features are observed in many CVD grown 3C$\mathrm{SiC} / \mathrm{Si}$ samples. Green's function calculations of impurity modes are used to model the defect structures that best supported the observed Raman features as well as modes of a prototypical center.

\section{Acknowledgements}

The authors wish to express their sincere thanks to Dr. J.A. Powell for the growth of the samples used in this study, to Drs. D. Pitt, K. Williams and K.T. Yue for the use of Raman instruments, and to Professors W.J. Choyke, I. Ferguson and C.C. Yang for their support and help throughout this project. The work of one of the authors (DNT) was supported by the Cottrell College Science Award \# CC4600 (Research Corporation), and by the National Science Foundation Grant No. ECS-9906077. 


\section{References}

[1] C.-M. Zetterling (Ed.), Process Technology for Silicon Carbide Devices, IEE Publishing, London, 2003.

[2] S.I. Vlaskina, Silicon carbide LED, Semiconductor Physics, Quantum Electronics \& Optoelectronics 5 (2002) 71.

[3] W.J. Choyke, M. Hiroyuki, G. Pensl (Eds.), Silicon Carbide: A Review of Fundamental Questions and Applications to Current Device Technology, Akademie Verlag GmbH, Berlin, 1997;

G.L. Harris (Ed.), Properties of Silicon Carbide, IEE Publishing, London, 1995.

[4] Z.C. Feng, J.H. Zhao (Eds.), Silicon Carbide: Materials, Processing and Devices, Taylor \& Francis, New York, 2003.

[5] C.C. Tin, R. Hu, R.L. Coston, J. Crystal Growth 148 (1995) 116.

[6] Z.C. Feng, A.J. Mascarenhas, W.J. Choyke, J.A. Powell, J. Appl. Phys. 64 (1988) 3176.

[7] P. Pirouz, C.M. Chorey, J.A. Powell, Appl. Phys. Lett. 50 (1987) 221.

[8] R.F. Davis, G. Kelner, M. Shur, J.W. Palmour, J.A. Edmond, Proc. of IEEE 79 (1991) 677.
[9] B. Segall, S.A. Alterovitz, E.J. Haugland, L.G. Matus, Appl. Phys. Lett. 49 (1986) 450, 584.

[10] A. Suzuki, A. Ogura, K. Furukawa, Y. Fujii, M. Shigeta, S. Nakajima, J. Appl. Phys. 64 (1988) 2818.

[11] W.J. Moore, J. Appl. Phys. 74 (1993) 1805;

W.J. Moore, J.A. Freitas, M.Yu. Altaiskii, V.L. Zuev, L.M. Ivanova, in: M.G. Spencer, R.P. Devaty, J.A. Edmond, M. Asif Khan, R. Kaplan, M. Rahman (Eds.), Inst. Phys. Conf. Ser. No. 137, 1994, p. 181.

[12] P. Pirouz, C.M. Chorey, J.A. Powell, Appl. Phys. Lett. 50 (1987) 221;

J.A. Powell, L.G. Matus, M.A. Kuczmarski, C.M. Chorey, T.T. Cheng, P. Pirouz, Appl. Phys. Lett. 51 (1987) 823.

[13] T. Chassagne, G. Ferro, C. Gourbeyre, M. Le Berre, D. Barbier, Y. Monteil, Mater. Sci. Forum 353 (2001) 155.

[14] Z.C. Feng, S.J. Chua, G.A. Evans, J.W. Steeds, K.P.J. Williams, G.D. Pitt, Mater. Sci. Forum 353 (2001) 345.

[15] Z.C. Feng, C.C. Tin, R. Hu, J. Williams, Thin Solid Films 266 (1995) 1.

[16] H.W. Shim, K.C. Kim, Y.H. Seo, K.S. Nahm, E.-K. Suh, H.J. Lee, Y.G. Hwang, Appl. Phys. Lett. 70 (1997) 1757.

[17] D.N. Talwar, M. Vandevyver, K.K. Bajaj, W.M. Theis, Phys. Rev. B 33 (1986) 8525. 